\title{
Study on the Influencing factors of the Nodal Dynamic Frequency Response Characteristics of Power System on Account of Curved Surface Fitting
}

\author{
Liang Shanshan ${ }^{1, a^{*}, \text { WU Jun }}{ }^{2, b}$, Liu Dichen ${ }^{3, \mathrm{c}}, \mathrm{Hu}$ Jingzhu $^{4, \mathrm{~d}}$,Han

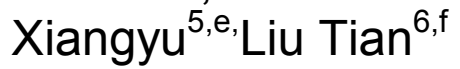

\author{
School of Electrical Engineering, Wuhan University, Luo-jia-shan Wuchang, Wuhan, Hubei \\ Province, 430072, China \\ alIs900305@163.com, bbyronwu@whu.edu.cn, ${ }^{\mathrm{c}}$ dcliu@whu.edu.cn, dijzhu_5@163.com,
egenhao3_han@163.com,LTLetitia@163.com
}

\begin{abstract}
Keywords: power systems stability; frequency characteristic coefficient; nodal dynamic frequency response characteristics; curved surface fitting

Abstract. Many studies on static frequency characteristics have been done yet nodal dynamic frequency response characteristics gain more attentions for the important role frequency plays in power system stability. Influencing factors such as balance power, power shortage and electrical distance are altered during PSASP simulation of IEEE36 bus system to acquire diverse sampling points and relevant $K$ values. Modified curved surface fitting obtained by the $K$ values can both be used to forecast $K$ values under any circumstances and simultaneously analyze the influencing effect. The method is proved to be feasible by analyzing the errors between simulation values and fitted values and it can offer reference for dispatch department to process the frequency variation caused by the power disturbance.
\end{abstract}

\section{Introduction}

Frequency is an important parameter to evaluate power quality and the safety and stability of power system. Currently, research on nodal dynamic frequency response characteristics of power system mainly focus on identification of failure node's frequency variation and system's average frequency variation in addition to calculation of static frequency characteristic ${ }^{[1-3]}$.

Static frequency characteristic records the quasi-steady values after transient subsidence, the maximum frequency deviation of which is related to the start of system emergency control measures, while the maximum of nodal dynamic frequency deviation depends on the operation mode and power balance before the system disturbance ${ }^{[4]}$. Actually frequency deviation of each node in the system which turns out to be merely of practical significance to slow process of frequency response is asynchronous during transient and it belongs to a rough range of values. As it is unable to reflect the nodal dynamic frequency response characteristics corresponding to different initial operation modes and states of disturbance rapidly, it can hardly be widely adopted in fast process such as system protection and emergency control ${ }^{[5]}$. Therefore theoretical and technical supports for identifying and predicting nodal frequency response characteristics adapting to multivariate power system are needed.

Certain influencing factors of observed dynamic frequency characteristics namely the initial operation mode, power shortage and electric distance are taken into consideration in the multivariate modified curved surface fitting proposed in this paper. Offline simulation is adopted to extract the finite sampling points which are necessary for the modified curved surface fitting. The errors between the simulation values and fitted values are calculated, which turns out that this method is of comparatively great value in theoretical and engineering application in that the rapidity, simplicity and accuracy of calculation are essentially improved compared with traditional recognition and calculation. 


\section{RESEARCH ON NODAL DYNAMIC FREQUENCY RESPONSE CHARACTERISTICS}

\section{Definition of nodal dynamic frequency response characteristics}

Nodal dynamic frequency response characteristics of power system refer to the ratio of power shortage $\Delta \mathrm{P}$ and system initial power $\mathrm{P} 0$ ratio and maximum frequency deviation $\Delta \mathrm{f}$ and system initial frequency $\mathrm{f} 0$ ratio when power shortage $\Delta \mathrm{P}$ breaks out at the observation space around observation points, frequency characteristic coefficient $\mathrm{K}$ of power system can be represented by the formula(1):

$$
K=\frac{\Delta P}{P_{0}} / \frac{\Delta f}{f_{0}}
$$

where $P_{0}$ and $f_{0}$ are respectively the power and frequency of system during normal operation.

Major factors such as initial balance power, power shortage and electrical distance are considered to study the effect on frequency characteristic coefficient.

Frequency characteristic coefficient $K$ is inversely proportional to the initial power $P_{0}$ which represents the initial operating state. The impact of disturbance on the system decreases as the initial power $P_{0}$ increases in that the regulating effect of system for disturbance increases.

It can be seen according to formula (1) that the frequency characteristic coefficient $K$ increases with the increasing of the power shortage $\Delta P$ which represents perturbation, on account that the impact of disturbances on power system is proportional to the disturbance quantity. Maximum frequency shift increases as power shortage increases, showing that the impact of disturbance on the system is larger.

The change of reactive power will lead to voltage change of the load point especially. Moreover, the voltage change will further affect the active power via voltage characteristics of the load which further affects the frequency characteristics of the power system accordingly. Electrical distance is an important indicator for evaluating temporal and spatial distribution characteristics.

Generally, the transfer reactance between two points is adopted to describe the electrical distance. It can be obtained by nodal impedance matrix indirectly which can be obtained by the following formula:

$$
Z_{f i}=\frac{\dot{E_{i}}}{\dot{I_{f i}}}=\frac{Z_{f f}}{Z_{f i}} z_{i}
$$

where $Z_{\mathrm{fi}}$ is transfer reactance, $Z_{\mathrm{ff}}$ is self-impedance observation points, $Z_{\mathrm{fi}}$ is the mutual impedance between observation point and disturbance point, $\mathrm{Z}_{\mathrm{i}}$ is the sub-transient impedance of disturbance point generator.

\section{FREQUENCY CHARACTERISTIC COEFFICIENT PREDICTION BASED ON CURVED SURFACE FITTING}

The method of obtaining $K$ values which is relatively accurate without affecting the normal operation of the power system is presented in this paper, namely the three-dimensional coordinate system $(K, S$, $\Delta P$ ) curved surface fitting corresponding to different balancing power respectively. $K$ values corresponding to any $\Delta P$ and $S$ can be predicted sequentially in addition.

\section{The main idea of the simulation PSASP}

Active disturbance is created by cutting generator appropriately and the maximum frequency deviation is recorded as $\Delta f$. $K$ values under different perturbations points corresponding to different $P_{0}$ and $\Delta P$ can be calculated by formula (1).

\section{Curved Surface Fitting}

Interpolation and fitting are required to handle data precisely and modify the rough curved surface fitted out by the finite data points obtained by PSASP to meet the desired $K$ values in case of different 
$\Delta P$ and $S$. The curved surface for nodal dynamic frequency response characteristics is based on cubic spline interpolation and fitting. Error analysis is necessary to verify the accuracy and precision.

\section{Frequency characteristic coefficient prediction based on Surface Fitting}

Curved surface of frequency characteristic coefficient $K$ and points of the surface correspond to $K$ values under different circumstances are obtained. It enables the identification and calculation of system frequency characteristic coefficient under multi-variable conditions to be realized. The curved three dimensional surface $(K, S, \Delta P)$ can provide reference for dispatch department when power disturbance bursts out.

\section{EXPERIMENTAL ANALYSIS AND SIMULATION OF NODE FREQUENCY CHARACTERISTIC AFFECTING FACTORS}

\section{Simulation Model Introduction}

EPRI-36 system one-line diagram adopted in the experiments is shown in Figure 1.

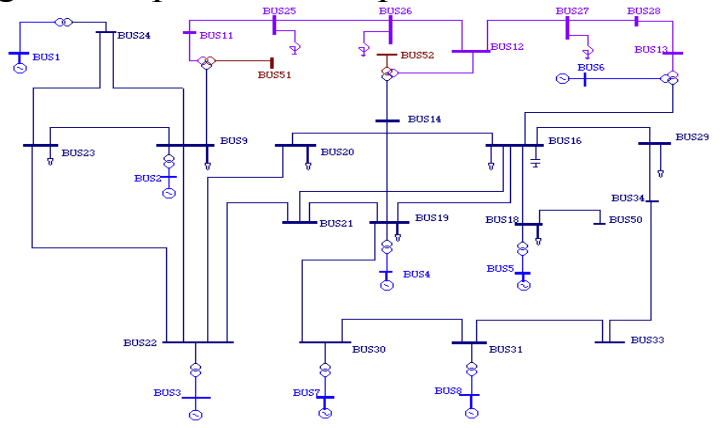

Figure 1. Pure AC system single-line diagram

The 1st current job is adopted in this experiment. Reference value of this model is 100[MVA]. BUS1 is regarded as balancing machine while BUS6 is generally considered to be phase modifier in practical application for its active output is minimal. It can be drawn by calculation that the total active power $P_{0}$ before disturbance is 26.5371 .

\section{Setting principles for the simulation variables}

Generator output and load should be reduced synchronously and proportionally as system's initial operational status changes. Both the variation of system's initial state and power shortage should be set appropriately.

The electric distance between observation point and disturbance center can be changed by cutting different generator after observation point is selected in the experiment.

\section{Simulation.}

BUS2 is selected as monitoring nodes in the experiment. It can be seen that the change in unit value of $\omega$ is the same with the change in unit value of frequency $\mathrm{f}$ according to formula $\omega=2 \pi \mathrm{f}$ when initial operating state, disturbance point and perturbation are confirmed. Therefore, the frequency change at BUS2 can be obtained indirectly by observing the angular velocity changes.

BUS3, BUS8 and BUS7 are selected as cutter points. The power shortage is set to be $0.1,0.2,0.3$ and 0.4. And the variation of the initial operation state is set to be 0.95 and 1.05 times.

The electrical distance between observation point and the disturbance point can be calculated by formula (2) as shown in Table 1according to the mathematical model, the generator and circuit parameters adopted in this simulation after the observation point and disturbance point are confirmed: 
Table 1. Electrical disturbances from the center to the detection point

\begin{tabular}{|l|l|l|}
\hline Disturbance center & Transfer impedance & Electrical distance (S) \\
\hline BUS3 & $0.0210+0.5076 \mathrm{i}$ & 0.5076 \\
\hline BUS4 & $0.0183+0.5685 \mathrm{i}$ & 0.5685 \\
\hline BUS5 & $0.0289+0.6010 \mathrm{i}$ & 0.6010 \\
\hline BUS8 & $0.0657+0.7503 \mathrm{i}$ & 0.7503 \\
\hline BUS7 & $0.0849+0.96995 \mathrm{i}$ & 0.9699 \\
\hline
\end{tabular}

The simulation results: Generator angular velocity curve at BUS2 is calculated by transient stability when disturbance point is set at BUS3 and perturbation is set to be 0.1 under the initial operating power. It can be learned from the data analysis according to formula (1) that angular velocity of the lowest point of the curve is 0.9973 , and it means that both $\Delta \omega$ and $\Delta \mathrm{f}$ is 0.0027 , therefore K value can be obtained to be 4.3266 .

The first set of data can be derived in the same way and calculation results are shown in Table 2.

Table 2. K value table with different disturbance point and disturbance when $P_{0}=26.5371$

\begin{tabular}{|l|c|c|l|l|l|l|l|l|l|}
\hline \multicolumn{2}{|c|}{$\Delta \mathbf{P}$} & \multicolumn{2}{c|}{$\mathbf{0 . 1}$} & \multicolumn{2}{c|}{$\mathbf{0 . 2}$} & \multicolumn{2}{c|}{$\mathbf{0 . 3}$} & \multicolumn{2}{c|}{$\mathbf{0 . 4}$} \\
\hline & $\boldsymbol{S}$ & $\omega$ & $\boldsymbol{K}$ & $\omega$ & $\boldsymbol{K}$ & $\omega$ & $\boldsymbol{K}$ & $\omega$ & $\boldsymbol{K}$ \\
\hline BUS3 & 0.508 & 0.997 & 4.327 & 0.996 & 5.169 & 0.993 & 5.467 & 0.992 & 5.631 \\
\hline BUS8 & 0.750 & 0.997 & 4.287 & 0.996 & 5.230 & 0.994 & 5.662 & 0.992 & 5.868 \\
\hline BUS7 & 0.970 & 0.998 & 3.837 & 0.997 & 4.831 & 0.995 & 5.355 & 0.994 & 5.653 \\
\hline
\end{tabular}

The output power of each generator and load decreased and increased by $5 \%$ respectively according to the above steps, and the disturbing quantity is set to be $0.1,0.2,0.3$ and 0.4 at BUS3, BUS8 and BUS7 respectively. Corresponding $K$ can be calculated in Table 3 and Table 4:

Table 3. K value table with different disturbance point and disturbance when $P_{0}=25.13854$

\begin{tabular}{|l|c|c|l|l|l|l|l|l|c|}
\hline \multicolumn{2}{|c|}{$\Delta \mathbf{P}$} & \multicolumn{2}{c|}{$\mathbf{0 . 1}$} & \multicolumn{2}{c|}{$\mathbf{0 . 2}$} & \multicolumn{2}{c|}{$\mathbf{0 . 3}$} & \multicolumn{2}{c|}{$\mathbf{0 . 4}$} \\
\hline & $\boldsymbol{S}$ & $\omega$ & $\boldsymbol{K}$ & $\omega$ & $\boldsymbol{K}$ & $\omega$ & $\boldsymbol{K}$ & $\omega$ & $\boldsymbol{K}$ \\
\hline BUS3 & 0.508 & 0.998 & 5.552 & 0.997 & 7.015 & 0.995 & 7.510 & 0.994 & 7.474 \\
\hline BUS8 & 0.750 & 0.998 & 5.586 & 0.997 & 7.183 & 0.956 & 7.814 & 0.994 & 7.815 \\
\hline BUS7 & 0.970 & 0.998 & 4.724 & 0.997 & 6.466 & 0.997 & 7.394 & 0.996 & 7.819 \\
\hline
\end{tabular}

Table 4. K value table with different disturbance point and disturbance when $P_{0}=27.96137$

\begin{tabular}{|l|c|c|l|l|l|l|l|l|c|}
\hline \multicolumn{2}{|c|}{$\Delta \mathbf{P}$} & \multicolumn{2}{c|}{$\mathbf{0 . 1}$} & \multicolumn{2}{c|}{$\mathbf{0 . 2}$} & \multicolumn{2}{c|}{$\mathbf{0 . 3}$} & \multicolumn{2}{c|}{$\mathbf{0 . 4}$} \\
\hline & $\boldsymbol{S}$ & $\omega$ & $\boldsymbol{K}$ & $\omega$ & $\boldsymbol{K}$ & $\omega$ & $\boldsymbol{K}$ & $\omega$ & $\boldsymbol{K}$ \\
\hline BUS3 & 0.508 & 0.995 & 2.461 & 0.993 & 3.485 & 0.991 & 3.978 & 0.989 & 4.085 \\
\hline BUS8 & 0.750 & 0.995 & 2.455 & 0.994 & 3.541 & 0.952 & 4.070 & 0.989 & 4.209 \\
\hline BUS7 & 0.970 & 0.996 & 2.012 & 0.995 & 3.061 & 0.993 & 3.695 & 0.992 & 4.062 \\
\hline
\end{tabular}

\section{FACTORS AFFECTING THE FREQUENCY CHARACTERISTICS OF THE NODES BASED ON SURFACE FITTING}

Frequency characteristic coefficient curved surfaces under different initial balance power can be obtained by substituting the data in Table 3 and 4 in the same way with cubic spline interpolation of Table 2.

\section{Fitting results}

Frequency characteristic coefficient fitting curves under different initial balance of power are shown in Figure 2: 

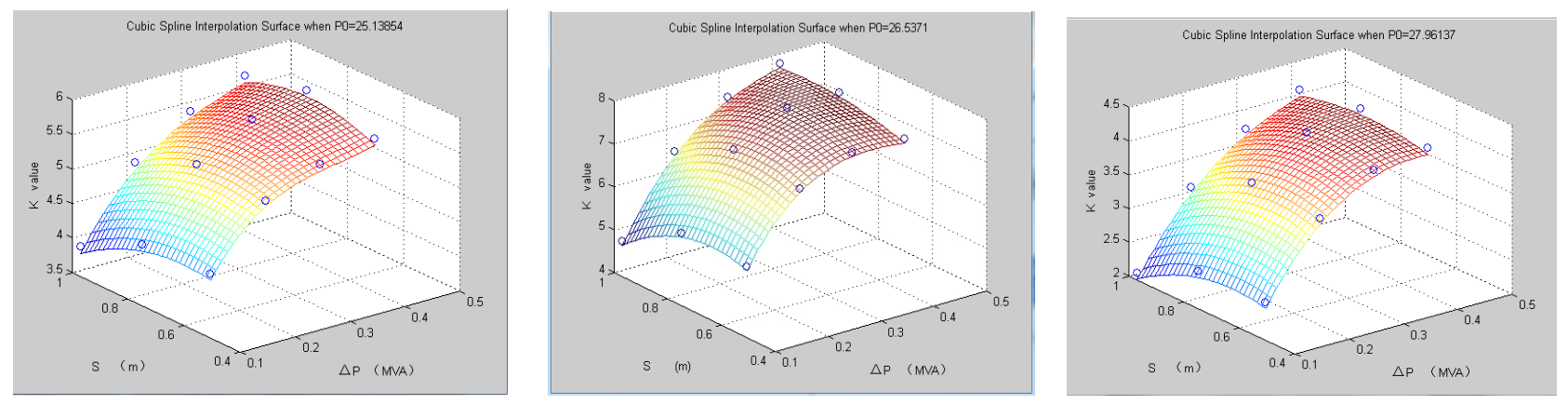

Figure 2. Cubic Spline Interpolation Surface when $P_{0}=25.13854 / 26.5371 / 27.9613$

Variation tendency of fitting surfaces: Surface change seems relatively smooth on the whole; the gradual upward trend is shown along X-axis which represents power disturbance; the tendency of decreasing firstly and then increasing is shown along $\mathrm{Y}$-axis which represents electrical distance $\mathrm{S}$ and the change is relatively flat; contrast three fitted surface longitudinally, vertical coordinates of the surface which represents the frequency characteristics coefficient $\mathrm{K}$ keep decreasing as the initial balance power continually increases and the steepness of the surface changes along X-axis increases.

Check of the accuracy of fitting results.

Surface is fitted by MATLAB in this paper based on the simulation values, error analysis is needed for the fitted out frequency characteristic coefficient surface to verify the accuracy and precision of the experiment. Error refers to the deviation between the fitting surface $K_{1}$ and simulation values $K_{1}^{\prime}$, as shown in formula (3):

$$
G=\frac{K_{1}-K_{1}^{\prime}}{K_{1}^{\prime}} \times 100 \%
$$

$K$ values corresponding to different $\Delta P$ are adopted to check the accuracy when $P_{0}$ was set to be 26.53710 and $S$ was set to be 0.600978 . $K$ values corresponding to different electrical distances are adopted to verify the accuracy again when $\Delta P$ was set to be 0.25 .

Accuracy is executed according to formula (3) and power reference value is $100 \mathrm{MW}$. Data can be obtained in Table 5, Table 6, Table 7, Table 8, Table 9 and Table 10:

Table 5. $K$ value of the check when $P_{0}=25.13854 S=0.600978$

\begin{tabular}{|c|c|c|c|}
\hline$\Delta \mathbf{P}$ & Simulation K value & Spline Fitting K value & Error \\
\hline 0.1 & 6.6598 & 5.6681 & $14 \%$ \\
\hline 0.2 & 8.0445 & 7.1987 & $11 \%$ \\
\hline 0.3 & 8.0445 & 7.7220 & $4.0 \%$ \\
\hline 0.4 & 7.6741 & 7.6459 & $0.4 \%$ \\
\hline
\end{tabular}

Table 6. $K$ value of the check when $P_{0}=25.13854 \Delta P=0.25$

\begin{tabular}{|c|c|c|c|}
\hline $\mathbf{S}$ & Simulation K value & Spline Fitting K value & Error \\
\hline 0.507623 & 7.3773 & 7.3560 & $0.29 \%$ \\
\hline 0.750302 & 7.6481 & 7.5978 & $0.66 \%$ \\
\hline 0.969869 & 6.9696 & 7.0123 & $0.61 \%$ \\
\hline
\end{tabular}

Table 7. $K$ value of the check when $P_{0}=26.5371 S=0.600978$

\begin{tabular}{|c|c|c|c|}
\hline$\Delta \mathbf{P}$ & Simulation K value & Spline Fitting K value & Error \\
\hline 0.1 & 5.0795 & 4.3681 & $14 \%$ \\
\hline 0.2 & 5.7974 & 5.359 & $9.4 \%$ \\
\hline 0.3 & 5.9792 & 5.254 & $6.2 \%$ \\
\hline 0.4 & 5.9627 & 5.7808 & $3.1 \%$ \\
\hline
\end{tabular}


Table 8. $K$ value of the check when $P_{0}=26.5371 \Delta P=0.25$

\begin{tabular}{|c|c|c|c|}
\hline S & Simulation K value & Spline Fitting K value & Error \\
\hline 0.507623 & 5.3488 & 5.3606 & $0.22 \%$ \\
\hline 0.750302 & 5.4910 & 5.4916 & $0.01 \%$ \\
\hline 0.969869 & 5.1200 & 5.1367 & $0.33 \%$ \\
\hline
\end{tabular}

Table 9. $K$ value of the check when $P_{0}=27096137 \quad S=0.600978$

\begin{tabular}{|c|c|c|c|}
\hline$\Delta \mathbf{P}$ & Simulation K value & Spline Fitting K value & Error \\
\hline 0.1 & 3.0751 & 2.5191 & $18 \%$ \\
\hline 0.2 & 4.1297 & 3.5796 & $13 \%$ \\
\hline 0.3 & 4.4771 & 4.0761 & $9 \%$ \\
\hline 0.4 & 4.3582 & 4.1682 & $4 \%$ \\
\hline
\end{tabular}

Table 10. $K$ value of the check when $P_{0}=27.96137 \quad \Delta P=0.25$

\begin{tabular}{|c|c|c|c|}
\hline $\mathbf{S}$ & Simulation K value & Spline Fitting K value & Error \\
\hline 0.507623 & 3.7943 & 3.7888 & $0.14 \%$ \\
\hline 0.750302 & 3.8715 & 3.8647 & $0.18 \%$ \\
\hline 0.969869 & 3.4179 & 3.4208 & $0.08 \%$ \\
\hline
\end{tabular}

\section{Result analysis.}

Errors between simulation values and fitted values are always less than $1 \%$ while electrical distance $S$ differs with the $P_{0}$ and $\Delta P$ keeping the same; The error increases with the $\Delta P$ decreasing, and it is less than $10 \%$ with larger $\Delta P$ on condition that the $P_{0}$ and $S$ are the same.

\section{Conclusion of fitting}

$K$ value increases with the $\Delta P$ increasing on condition that $P_{0}$ and $S$ stay the same. It means that the impact of the disturbances on the power system increases with the disturbance increasing; Electrical distance has no distinct effect on the frequency characteristics coefficient, for what has been adopted is 36 nodes simple power system hence electrical distance can vary within fairly limited range; The frequency characteristic coefficient increases with $P_{0}$ increasing when $\Delta P$ and $S$ stay the same. It means that the impact of the same disturbances on power system decreases when $P_{0}$ increases.

\section{CONCLUSION}

Frequency response characteristic coefficients are obtained by curved surface fitting in this paper; conclusions can be drawn as follow:

The method of obtaining frequency response characteristic coefficient of power system by adopting curved surface fitting is feasible and the simulation result which reflects the actual $K$ values on condition that the errors are within permissible range is relatively desirable;

The relationship between the node dynamic frequency response characteristics and its influencing factors can be reflected intuitively by the curved surface and the correctness of theoretical analysis is verified;

The curved surface in this paper is of great reference value when $K$ value of the power system is needed to adjust load in practical operation in case of emergency, it can offer reference for dispatch department to process the frequency variation caused by the power disturbance;

Method of reversing sampling points can be adopted to forecast change trend of nodal dynamic frequency response characteristics, frequency deviation and change of frequency stability.

Meanwhile certain improvements are needed in this paper:

Node model adopted for simulation in this paper is EPRI-36, nevertheless it is different from the real power system, wherefore more complex node model may be adopted when condition permits. More surface fitting methods should be adopted for data processing so that more suitable curved surface fitting methods can be chosen. 


\section{Acknowledgement:}

This paper is based on the project of Natural Science Foundation Funded projects : 51207114

\section{References}

[1] Guangming LU, Junli CUI, Shujun YAO, XU Lie. Automation of Electric Power Systems. 2008, 32(01): 29-33. (In Chinese)

[2] Qiang ZHAO, Limin WANG, Zhaoxu LIU, Guang-quan BU. Power System Technology, 2009, 33(08): 35-40. (In Chinese)

[3] Haifeng ZHOU, Laqin NI, Taishan XU. Power System Technology, 2009, 33(16): 58-62. (In Chinese)

[4] Jun WU, Dichen LIU.. Power System Protection and Control, 2012, 40(06): 97-103. (In Chinese)

[5] Feifei Dong, Dichen Liu, Jun,Zhang, Lin Wu, Lei Yun, Xiaoming Wang, Rusong Wang. Electric Power Automation Equipment, 2012, 32(07): 104-107. (In Chinese)

[6] Li C F, Cheng R J H. A two-stage digital AGC scheme with diversity selection for frame based OFDM systems [A]. In: Proceedings of 2006 IEEE International Symposium on Circuits and Systems (ISCAS2006XC), May21-24, 2006, Kos, Greece. Piscataway, NJ, USA, 2006: 3530-3533.

[7] Aimin Li, Zexiang Cai. TRANSACTIONS OF CHINA ELECTROTECHNICAL SOCIETY. 2009, 24(09): 171-177. (In Chinese)

[8] Maohai Wang, Zhengshan Xu, Kai Xie, Shaokun Lv. Automation of Electric Power Systems, 2007, 31(03): 15-18, 46.. (In Chinese)

[9] Gillian L, Alan M, Malley M O. Frequency control andwind turbine technologies [J]. IEEE Transactions on PowerSystems, 2005, 20(4): 1905-1913.

[10]Hengxu Zhang, Changgang Li, Yutian,Huang Liu, long Zhi, Kanqin Zhuang. TRANSACTIONS OF CHINA ELECTROTECHNICAL SOCIETY, 2010, 25(11): 169-176. (In Chinese)

[11] Weihua ZHANG, Jinsha YUAN, Tiefeng ZHANG, Ke ZHANG. Proceedings of the CSEE, 2014, 34(24): 4129-4136. (In Chinese)

[12]Jun YI, Xiaoxin ZHOU. Automation of Electric Power Systems, 2006, 30(14): 1-5. (In Chinese)

[13] Yu Lei.Factors affecting the modern power system dynamic frequency characteristics.Chong Qing university.2002 (In Chinese)

[14] Wenlue Chen, Ziyang Wang. JOURNAL OF CENTRAL CHNA NORMAL UNIVERSITY ,2004, 38(4): 54-58 (In Chinese) 